Short Communication

\title{
Separation of Water-Insoluble and Neutral Linear Synthetic Polymers in Nonaqueous Capillary Zone Electrophoresis Using Phosphonium-Based Ionic Liquid
}

\author{
Nao FUKAI, Shinya KITAGAWA*, Hajime OHTANI \\ Department of Life Science and Applied Chemistry, Graduate School of Engineering, Nagoya Institute of Technology,
}

Gokiso, Showa, Nagoya 466-8555, Japan

\begin{abstract}
The separation of neutral and water-insoluble linear synthetic polymers in non-aqueous capillary zone electrophoresis using the hydrophobic phosphonium-based ionic liquid trihexyl(tetradecyl)phosphonium chloride $\left(\mathrm{P}_{66614} \mathrm{Cl}\right)$ were investigated using polystyrene, polymethylmethacrylates, polybutadiene, and polycarbonate as test analytes. The solubility of $\mathrm{P}_{66614} \mathrm{Cl}$ in tetrahydrofuran was above $500 \mathrm{mM}$, which was significantly higher than that of the general ionic surfactants used in our previous studies. The separation of the four polymers was achieved using a binary mixture of tetrahydrofuran/methanol or tetrahydrofuran/acetonitrile containing $\mathrm{P}_{66614} \mathrm{Cl}$ as the electrophoretic medium. It was confirmed that an increase in hydrophobicity or bulkiness of the cationic reagent improved the polymer separation. The interaction between polycarbonate with $\mathrm{P}_{66614} \mathrm{Cl}$ was reduced by the addition of acetonitrile, and enhanced by the methanol addition.
\end{abstract}

Keywords: Ionic liquid; Nonaqueous capillary zone electrophoresis; Selectivity; Synthetic polymers

\section{Introduction}

High performance liquid chromatography (HPLC) is frequently used for separating and characterizing both synthetic polymers and biopolymers [1-3]. Electrophoresis is an alternative separation method and often applied to the analysis of biopolymers such as proteins and DNAs [4-8]. However, in contrast to biopolymers, electrophoretic methods have rarely been applied to the synthetic polymer analysis, whereas the electrophoretic method has a potential to achieve separations with different selectivity compared to HPLC. Moreover, the volume of electrophoretic medium required for capillary electrophoresis (CE) is significantly smaller than that for conventional HPLC. Therefore, development of a CE method for synthetic polymers is highly desirable in terms of both cost and environmental impacts.

CE separations are typically performed in aqueous media and have been applied to water-soluble and charged synthetic oligomers and polymers [9-13]. In addition,

${ }^{*}$ Corresponding author: Shinya KITAGAWA

Tel: +81-52-735-5368; Fax: +81-52-735-5368

E-mail: kitagawa.shinya@nitech.ac.jp nonaqueous (NA) capillary electrophoresis (NACE) has been applied to water-insoluble and charged oligomers and polymers $[10,14,15]$. Notably, many synthetic polymers, such as polystyrenes (PSs) and polymethylmethacrylates (PMMAs), are water-insoluble and neutral in solution. Therefore, only a few studies have focused on their CE separation, and the study of their electrophoretic behavior is insufficient [16-18].

In 2004, Li et al. reported the size-based separation of PSs and PMMAs of different molecular weights in nonaqueous capillary gel electrophoresis (NACGE) with a dimethylformamide-based electrophoretic medium containing polyethylene oxide as the sieving matrix and stearyltributylphosphonium bromide as the source of electrophoretic mobility for the neutral polymer analyte [16]. Recently, we demonstrated the separation of four water-insoluble and neutral linear synthetic polymers (PS, PMMA, polybutadiene (PBD), and polycarbonate (PC)) in nonaqueous capillary zone electrophoresis (NACZE) using

Received: 28 April 2017

Accepted: 24 June 2017

J-STAGE Advance Published: 2 July 2017

DOI: 10.15583/jpchrom.2017.008 
tetrahydrofuran (THF)-based electrophoretic medium containing cetyltrimethylammonium chloride (CTAC) [17]. In contrast to NACGE, the separation depended on only the polymer species, i.e., independence on the molecular size distribution of the polymers.

Further study revealed that the separation of neutral polymers in NACZE using ionic surfactants is affected by the hydrophobic interactions between the polymers and the ionic surfactants [18]. The addition of a relatively polar solvent, such as acetonitrile (ACN) or water, to the electrophoretic medium enhances the hydrophobic interactions between polymers and surfactants, but often results in decrease in polymer solubility to the medium. On the other hand, the addition of a polar solvent such as alcohol or water to the NA medium is often required to dissolve the ionic surfactant in the medium, because THF, a general solvent for synthetic neutral polymers, is typically a poor solvent for ionic surfactants. In our previous study, the mixture of three solvents (THF and other two solvents of $\mathrm{ACN}$, ethanol, methanol $(\mathrm{MeOH})$, or water) was necessary for the separation of PS, PMMA, PBD, and PC, and the optimization procedure was complex and time-consuming.

Ionic liquids (ILs) are salts that are liquid state at relatively low temperatures [19], and have often been used as additives to electrophoretic media to control the electrophoretic behavior. Phosphonium-based ILs have relatively low absorption in the UV and visible regions, and have been used as the pseudostationary phase in electrokinetic chromatography with UV absorbance detection [20,21]. Highly hydrophobic phosphonium-based ILs have high solubility in THF, and can therefore be used as the additive to THF-based NA solvent for providing a driving force for electrophoretic migration of the neutral polymers because, as described above, ionic surfactants interact hydrophobically with polymers. Moreover, in our previous paper, we predicted that the use of highly hydrophobic cation would be suitable for the polymer separation [18].

In this study, the use of the highly hydrophobic phosphonium-based IL trihexyl(tetradecyl)phosphonium chloride $\left(\mathrm{P}_{66614} \mathrm{Cl}\right)$ was investigated for the NACZE separation of water-insoluble and neutral linear synthetic polymers. For the comparison with the previous results, the same polymers, namely PS, PMMA, PBD, and PC, were used as test analytes. The effect of the NA medium composition on the electrophoretic behavior was also investigated using simple binary mixtures of $\mathrm{THF} / \mathrm{MeOH}$ and THF/ACN as electrophoretic media.

\section{Experimental}

The apparatus and procedure are similar to those described in our previous study [18]. A commercial CAPI-3100 instrument (Otsuka electronics, Osaka, Japan)
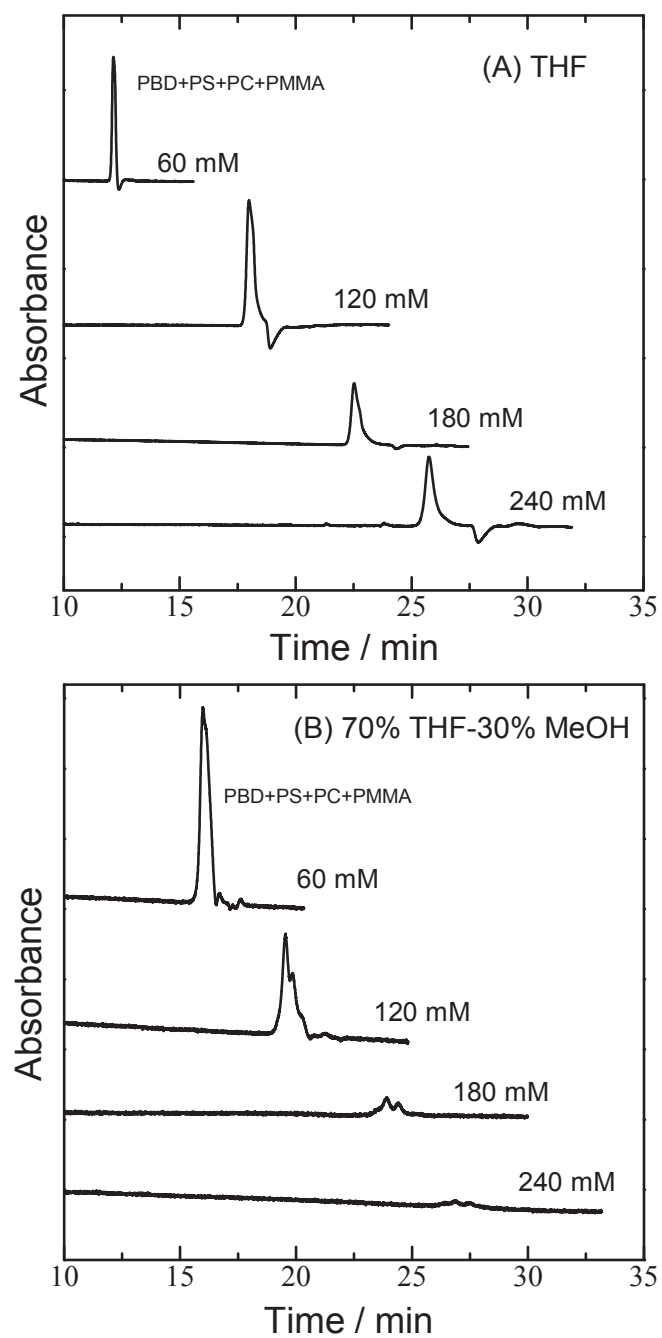

Fig. 1 Effect of $\mathrm{P}_{66614} \mathrm{Cl}$ concentration on separation of four synthetic polymers (PS, PMMA, PBD, and PC) in NACZE using (A) THF and (B) THF containing $30 \% \mathrm{MeOH}$ as electrophoretic media. $\mathrm{P}_{66614} \mathrm{Cl}$ concentration is given beside each electropherogram. Capillary: $44 / 56 \mathrm{~cm}$, i.d. $0.05 \mathrm{~mm}$; voltage: +25 $\mathrm{kV}$; detection: $220 \mathrm{~nm}$.

with a fused-silica capillary of i.d. $0.050 \mathrm{~mm}$ with inert treatment (whole length: $56 \mathrm{~cm}$, effective length: $44 \mathrm{~cm}$, GL Sciences, Tokyo, Japan) was used. Separations were carried out with the application of $+25 \mathrm{kV}$ at $25{ }^{\circ} \mathrm{C}$, and the analytes were detected by UV absorbance at $220 \mathrm{~nm}$. Prior to each run, the capillary was sequentially rinsed with THF (300 s), ethanol (300 s), and the electrophoretic medium (300 s) by vacuum. The sample solution was injected by a hydrodynamic method $(\Delta H=25 \mathrm{~mm}, 30 \mathrm{~s})$. Because our NACZE separation often requires a longer migration time, the CE separation was performed with a slightly pressurized flow toward the outlet, i.e., the liquid level of the inlet buffer was $1 \mathrm{~cm}$ higher than that of the outlet buffer.

THF, ACN, MeOH, and ethanol were purchased from Wako Pure Chemicals (Osaka, Japan). $\mathrm{P}_{66614} \mathrm{Cl}$ was purchased from Sigma-Aldrich (St. Louis, MO, USA). 
Polymers of PS $\left(\mathrm{M}_{\mathrm{n}}=6500\right.$, Showa Denko, Tokyo, Japan), $\operatorname{PBD}\left(\mathrm{M}_{\mathrm{n}}=5900\right.$, Sowa Science), PMMA $\left(\mathrm{M}_{\mathrm{n}}=525,000\right.$, Wako), and PC $\left(M_{n}=6700\right)$ were used as analytes.

\section{Results and discussion}

\subsection{Effect of ionic liquid concentration}

In our previous research, we demonstrated the separation of neutral linear synthetic polymers by NACZE using three-solvent mixtures containing an ionic surfactant (CTAC, dimethyldioctadecylammonium bromide (DMDOAB), or sodium dodecylsulfate (SDS)) [17,18]. As reported in our previous paper, the solubility of CTAC, DMDOAB, and SDS in THF is lower than $1 \mathrm{mM}$ [18]. In this study, the solubility of $\mathrm{P}_{66614} \mathrm{Cl}$ was measured to be over $500 \mathrm{mM}$ in $\mathrm{THF}$ as well as $\mathrm{MeOH}$ and $\mathrm{ACN}$ at room temperature.

Because of the high solubility of $\mathrm{P}_{66614} \mathrm{Cl}$ in THF, separation of the four polymers (PS, PMMA, PBD, and PC) was investigated using pure THF containing different concentrations of $\mathrm{P}_{66614} \mathrm{Cl}$ as the electrophoretic medium, and obtained electropherograms are shown in Fig. 1A. An increase in the $\mathrm{P}_{66614} \mathrm{Cl}$ concentration resulted in an increase in elution time; however, in any cases, a single peak was observed. That is, the four polymers could not be separated under these conditions.

The effect of the $\mathrm{P}_{66614} \mathrm{Cl}$ concentration in a THF/MeOH mixture (70/30) on the electrophoretic separation was also investigated, as shown in Fig. 1B. In this case, $60 \mathrm{mM}$ $\mathrm{P}_{66614} \mathrm{Cl}$ resulted in an almost single peak, indicating a co-migration of the four polymers similar to Fig. 1A. In contrast, an increase in $\mathrm{P}_{66614} \mathrm{Cl}$ concentration improved the separation of the polymers. However, the peak height of the polymer significantly decreased and almost no peak was observed at $240 \mathrm{mM}$. That is, the higher $\mathrm{P}_{66614} \mathrm{Cl}$ concentration enhanced the separation performance, but decreased the sensitivity, which would be provided mainly by an increase in the background absorbance. As can be seen from Fig. 1B, the maximum acceptable $\mathrm{P}_{66614} \mathrm{Cl}$ concentration was approximately $180 \mathrm{mM}$.

In this study, a EOF marker suitable for various NA conditions was not found and some experimental conditions resulted in relatively unstable migration times of the polymers whereas the elution order were reproduced. Therefore, in the following sections, we focused on the discussion about the elution order (or selectivity) only.

\subsection{Effect of mobile phase composition}

A MeOH addition to a THF-based medium is effective in enhancing polymer separation as shown in Fig. 1. Therefore, the influence of NA medium composition on polymer separation was investigated, and the electropherograms obtained using electrophoretic media containing $180 \mathrm{mM}$ $\mathrm{P}_{66614} \mathrm{Cl}$ and different $\mathrm{MeOH}$ concentrations are shown in
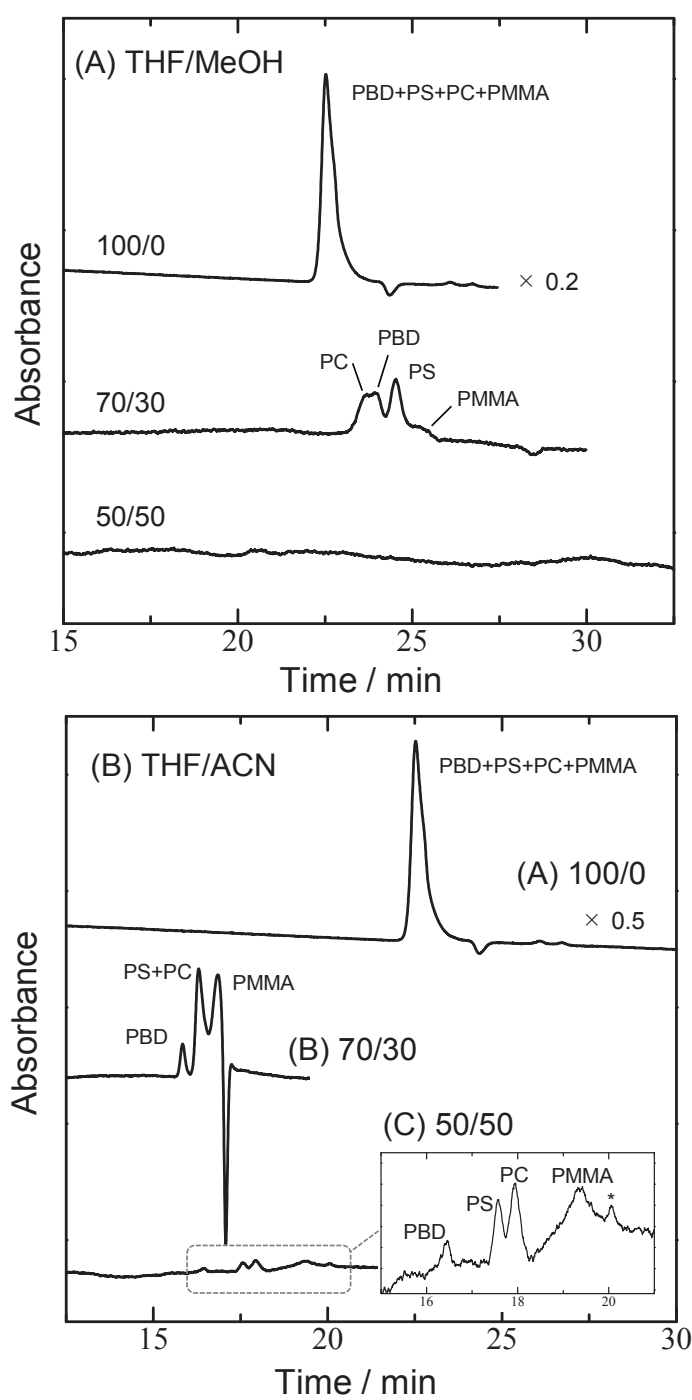

Fig. 2 Effect of the composition of the nonaqueous medium on separation of four synthetic polymers (PS, PMMA, PBD, and PC) in NACZE using THF mixed with (A) $\mathrm{MeOH}$ and (B) ACN containing $180 \mathrm{mM} \mathrm{P}{ }_{66614} \mathrm{Cl}$. The peaks marked with $*$ is unknown. Other conditions are the same as in Fig. 1.

Fig. 2A. Although the peaks are partially overlapped, the four polymers were separated when a THF-based electrophoretic medium containing $30 \% \mathrm{MeOH}$ was used. However, the electropherogram obtained using a medium containing $50 \% \mathrm{MeOH}$ did not show significant peaks. Further $\mathrm{MeOH}$ addition was not beneficial to a detection. A similar decrease in the UV absorbance signal was also observed with THF/ACN mixtures, as shown in Fig. 2B. However, in this case, the polymer peaks were detected when a THF-based medium containing $50 \% \mathrm{ACN}$ was used.

As shown in Fig. 2B, increasing the ACN concentration clearly improved the separation. The four polymers were separated using a medium containing $50 \% \mathrm{ACN}$, whereas PC and PS were co-eluted when a medium containing 30\% $\mathrm{ACN}$ was used. Because a positive voltage was applied at 
Table 1. Effect of additive species and composition of the electrophoretic medium on the selectivity

\begin{tabular}{|c|c|c|c|c|}
\hline \# & Additive & $\mathrm{mM}$ & Composition & Interaction order \\
\hline $1^{\text {a) }}$ & CTAC & 70 & $\begin{array}{l}\text { THF/MeOH } \\
70 / 30\end{array}$ & not separated \\
\hline $2^{\text {a) }}$ & & 70 & $\begin{array}{l}\text { THF/ACN/MeOH } \\
50 / 40 / 10\end{array}$ & PBD $>$ PC $>$ PS $>$ PMMA \\
\hline $3^{\text {a) }}$ & & 70 & $\begin{array}{l}\mathrm{THF} / \mathrm{MeOH} / \mathrm{H}_{2} \mathrm{O} \\
68 / 29 / 3\end{array}$ & PC $>$ PBD $>$ PS $>$ PMMA \\
\hline $4^{\text {a) }}$ & DMDOAB & 30 & $\begin{array}{l}\text { THF/MeOH } \\
70 / 30\end{array}$ & $\mathrm{PC}>\mathrm{PBD}>\mathrm{PS}=\mathrm{PMMA}$ \\
\hline $5^{\text {a) }}$ & & 40 & $\begin{array}{l}\mathrm{THF} / \mathrm{ACN} / \mathrm{MeOH} \\
50 / 10 / 40\end{array}$ & PBD $>$ PC $>$ PS $>$ PMMA \\
\hline 6 & $\mathrm{P}_{66614} \mathrm{Cl}$ & 180 & $\begin{array}{l}\mathrm{THF} / \mathrm{MeOH} \\
70 / 30\end{array}$ & PC $>$ PBD $>$ PS $>$ PMMA \\
\hline 7 & & 180 & $\begin{array}{l}\text { THF/ACN } \\
70 / 30\end{array}$ & $\mathrm{PBD}>\mathrm{PS}=\mathrm{PC}>\mathrm{PMMA}$ \\
\hline 8 & & 180 & $\begin{array}{l}\mathrm{THF} / \mathrm{ACN} \\
50 / 50\end{array}$ & $\mathrm{PBD}>\mathrm{PS}>\mathrm{PC}>\mathrm{PMMA}$ \\
\hline
\end{tabular}

a) Our previous results reported in reference 18 .

the capillary inlet, the polymer strongly interacting with $\mathrm{P}_{66614}$ cation were detected earlier. The interaction order, or selectivity, is summarized in Table 1, which also includes the results of our previous work for comparison [18]. Addition of CTAC to the THF/MeOH (70/30) medium failed to separate the polymers (\#1 in Table 1), whereas the DMDOAB addition resulted in partial separation (\#4). The use of $\mathrm{P}_{66614} \mathrm{Cl}$ achieved a separation of the four polymers (\#6). Thus, an increase in hydrophobicity or bulkiness of the cationic reagent was found to improve the separation. The prediction in our previous study is successfully confirmed, whereas their electrophoretic conditions, in particular for the additive concentration, were different. However, the detail of the effect of hydrophobicity or bulkiness of the cationic reagent on polymer selectivity (difference in the interaction between the polymer and the regent) is not clarified yet. Further quantitative studies are necessary for elucidating the electrophoretic behavior.

As shown in Table 1, DMDOAB and $\mathrm{P}_{66614} \mathrm{Cl}$ gave almost the same selectivity (\#4 and \#6). In our previous study, we have reviled that the interaction order of PBD, PS, and PMMA was common in the separations using CTAC, DMDOAB, and SDS, which is related with the elution order of the polymers in gradient reversed-phase HPLC $[17,18]$. This order was also found using $\mathrm{P}_{66614} \mathrm{Cl}$ in both $\mathrm{THF} / \mathrm{MeOH}$ and THF/ACN mixtures. On the other hand, the interaction of $\mathrm{PC}$ with $\mathrm{P}_{66614}$ cation was clearly affected by the medium composition. Namely, the interaction between $\mathrm{PC}$ with $\mathrm{P}_{66614} \mathrm{Cl}$ was reduced by the addition of $\mathrm{ACN}$ and enhanced by $\mathrm{MeOH}$ addition. The difference in the behaviors may relate with the difference in hydrogen bonding property between ACN and $\mathrm{MeOH}$. However, detailed study is required to clarify this difference. Although similar behaviors might been observed for CTAC (\#2 and \#3) and DMDOAB (\#4 and \#5), the use of three-solvent mixture complicated the behavior. On the other hand, the combination of simple binary mixtures and highly hydrophobic cation was demonstrated to be a powerful technique to study the NACZE behavior of polymers.

\section{Acknowledgement}

This work was supported by JSPS KAKENHI Grant Number 15K13723. We would like to express our gratitude to Dr. Sueyoshi (Osaka Prefecture University) for his helpful suggestion about ILs.

\section{References}

[1] HPLC of Polymers; Pasch, H.; Trathnigg, B.; Springer-Verlag: Berlin, Heidelberg; 1999.

[2] HPLC of Peptides and Proteins Basic Theory and Methodology; Aguilar, M.-I. Ed.; Humana Press: Totawa, NJ, 2004.

[3] Katz, E. D. HPLC of DNA and PCR Products. In The Nucleic Acid Protocols Handbook; Rapley, R. Ed; Humana Press: Totawa, NJ, 2004; pp 105-113.

[4] Huck, C. W.; Bonn, G. K. Analysis of Proteins by Capillary Electrophoresis. In Capillary Electrophoresis: Methods and Protocols; Kopplin, P. S., Ed.; Springer-Verlag: Berlin Heidelberg, 2008; pp 507-540.

[5] Geiger, M.; Hogerton, A. L.; Bowser, M. T. Anal. Chem. 2012, 84, 577-596.

[6] Zhu, Z.; Lu, J. J.; Liu, S. Anal. Chim. Acta 2012, 709, 21-31.

[7] Righetti, P. G.; Sebastiano, R.; Citterio, A. Proteomics 2013, 13, 325-340.

[8] McCord, B.; Hartzell-Baguley, B.; King, S. Separation of DNA by Capillary Electrophoresis. In Capillary Electrophoresis: Methods and Protocols; Kopplin, P. S., Ed.; Springer-Verlag: Berlin Heidelberg 2008; pp 415-429.

[9] Starkweather, M. E.; Hoagland, D. A.; Muthukumar, M. Macromolecules 2000, 33, 1245-1253.

[10] Cottet, H.; Simo, C.; Vayaboury, W.; Cifuentes, A. J. Chromatogr. A 2005, 1068, 59-73.

[11] Cottet, H.; Gareil, P. Separation of Synthetic (Co)Polymers by Capillary Electrophoresis Techniques. In Capillary Electrophoresis: Methods and Protocols; Kopplin P. S., Ed.; Springer-Verlag: Berlin, Heidelberg 2008; pp 541-567.

[12] Lefay, C.; Guillaneuf, Y.; Moreira, G.; Thevarajah, J. J.; Castignolles, P.; Ziarelli, F.; Bloch, E.; Major, M.; Charles, L.; Gaborieau, M.; Bertin, D.; Gigmes, D. Polym. Chem. 2013, 4, 322-328.

[13] Thevarajah, J. J.; Gaborieau, M.; Castignolles, P. Advances in Chemistry 2014, Article ID 798503, 11 pages.

[14] Jansson, M.; Roeraade J. Chromatographia 1995, 40, 163-169.

[15] Cottet, H.; Struijk M. P.; Van Dongen, J. L.; Claessens, 
H. A.; Cramers, C. A. J. Chromatogr. A 2001, 915, 241-251.

[16] Li, G.; Zhou, X.; Wang, Y.; Krull, I. S.; Mistry, K.; Grinberg, N.; Cortes, H. J. Liq. Chromaogr. Relat. Technol. 2004, 27, 939-964.

[17] Yamamura, T.; Kitagawa, S.; Ohtani, H. J. Chromatogr. A 2015, 1393, 122-127.

[18] Fukai, N.; Kitagawa, S.; Ohtani, H. Electrophoresis 2017, 38, 1724-1729.

[19] Ionic Liquids, Kirchner, B., Ed.; Springer-Verlag: Berlin, Heidelberg, 2009.

[20] Wiedmera, S. K.; Kingb, A. W. T.; Riekkolaa, M. J. Chromatogr. A 2012, 1253, 171-176.

[21] Krízek, T.; Breitbach, Z. S.; Armstrong, D. W.; Tesarová, E.; Coufal, P. Electrophoresis 2009, 30, 3955-3963. 